\title{
Blood Accessibility to Fibrin in Venous Thrombosis is Thrombus Age-Dependent and Predicts Fibrinolytic Efficacy: An In Vivo Fibrin Molecular Imaging Study
}

\author{
Ashley F. Stein-Merlob ${ }^{1^{*}}$, Chase W. Kessinger ${ }^{*}$, S. Sibel Erdem ${ }^{2,6}$, Henry Zelada ${ }^{1}$, Scott A. Hilderbrand ${ }^{2}$, \\ Charles P. Lin 2,3, Guillermo J. Tearney33, Michael R. Jaff'1, Guy L. Reed", Peter K. Henke5, Jason R. \\ McCarthy ${ }^{2 \dagger}$, Farouc A. Jaffer $1,3+\bowtie$ \\ 1. Cardiovascular Research Center, Division of Cardiology, Massachusetts General Hospital, Harvard Medical School \\ 2. Center for Systems Biology, Massachusetts General Hospital, Harvard Medical School \\ 3. Wellman Center for Photomedicine, Massachusetts General Hospital, Harvard Medical School \\ 4. Department of Medicine, University of Tennessee Health Sciences Center \\ 5. Section of Vascular Surgery, Department of Surgery, University of Michigan, Ann Arbor, MI \\ 6. Current Address: Istanbul Medipol University \\ *AFS and CWK contributed equally \\ ${ }^{\dagger}$ FAJ and JRM share senior authorship \\ $\triangle$ Corresponding author: Farouc Jaffer MD PhD FAHA, MGH CVRC, 185 Cambridge Street, Simches Research Building, Room 3206, Boston, Massachusetts \\ 02114. E-mail: fjaffer@mgh.harvard.edu; Tel: (617) 724-9353; Fax: (617) 860-3180
}

(C) 2015 Ivyspring International Publisher. Reproduction is permitted for personal, noncommercial use, provided that the article is in whole, unmodified, and properly cited. See http://ivyspring.com/terms for terms and conditions.

Received: 2015.04.23; Accepted: 2015.08.07; Published: 2015.09.13

\begin{abstract}
Fibrinolytic therapy of venous thromboembolism (VTE) is increasingly utilized, yet limited knowledge is available regarding in vivo mechanisms that govern fibrinolytic efficacy. In particular, it is unknown how age-dependent thrombus organization limits direct blood contact with fibrin, the target of blood-based fibrinolytic agents. Utilizing high-resolution in vivo optical molecular imaging with FTP1 1, a near-infrared fluorescence (NIRF) fibrin-specific reporter, here we investigated the in vivo interrelationships of blood accessibility to fibrin, thrombus age, thrombus neoendothelialization, and fibrinolysis in murine venous thrombosis (VT). In both stasis VT and non-stasis VT, NIRF microscopy showed that FTPII fibrin binding was thrombus age-dependent. FTP11 localized to the luminal surface of early-stage VT, but only minimally to subacute VT $(p<0.001)$. Transmission electron microscopy of early stage VT revealed direct blood cell contact with luminal fibrin-rich surfaces. In contrast, subacute VT exhibited an encasing CD31+ neoendothelial layer that limited blood cell contact with thrombus fibrin in both VT models. Next we developed a theranostic strategy to predict fibrinolytic efficacy based on the in vivo fibrin accessibility to blood NIRF signal. Mice with variably aged VT underwent FTPI 1 injection and intravital microscopy (IVM), followed by tissue plasminogen activator infusion to induce VT fibrinolysis. Fibrin molecular IVM revealed that early stage VT, but not subacute VT, bound FTPII $(p<0.05)$, and experienced higher rates of fibrinolysis and total fibrinolysis $(p<0.05$ vs. subacute $V T)$. Before fibrinolysis, the baseline FTP1 1 NIRF signal predicted the net fibrinolysis at 60 minutes $(p<0.001)$. Taken together, these data provide novel insights into the temporal evolution of VT and its susceptibility to therapeutic fibrinolysis. Fibrin molecular imaging may provide a theranostic strategy to identify venous thrombi amenable to fibrinolytic therapies.
\end{abstract}

Key words: Fibrin; fibrinolysis; venous thrombosis; molecular imaging; pulmonary embolism

\section{Introduction}

Venous thromboembolism (VTE) is a major cause of morbidity and mortality worldwide. Fibrinolytic therapy mediated by blood-based plasmino- gen activators, either systemic or catheter-directed, can effectively reduce morbid VTE complications including cardiovascular collapse and the 
post-thrombotic syndrome. $(1,2)$ However, not all VT respond favorably to fibrinolytic therapy, which also carries a significant risk of life-threatening hemorrhage.(3) While clinical studies link older thrombus age to less successful fibrinolysis, and suggest binary age thresholds for administering fibrinolytic therapy, $(3,4)$ remarkably little knowledge is available regarding in vivo mechanisms underlying these observations. Therefore, theranostic studies that provide mechanistic insights into the age-dependent decrease in VT fibrinolysis, as well identify VT likely to respond to fibrinolysis, could significantly improve the benefit-to-risk ratio of fibrinolytic therapies.

As younger venous thrombi are often more amenable to fibrinolysis $(3,4)$ and aging thrombi undergo neovascularization as part of VT resolution(5), we hypothesized that blood accessibility to fibrin, the protein target of plasminogen activators, would be greater in younger aged thrombi, and that older thrombi would show reduced fibrin accessibility related to the development of a luminal thrombus neoendothelial layer. Furthermore, we hypothesized the degree of blood accessibility to fibrin would predict the ability of blood-based fibrinolytic agents to lyse VT. In this in vivo experimental study, we harnessed intravital microscopy (IVM) and FTP11 (6), a near-infrared fluorescence (NIRF) fibrin-specific molecular imaging reporter, to investigate mechanisms of fibrin accessibility in vivo. First we assessed age-dependent binding of FTP11 to fibrin in stasis and non-stasis VT, and then assessed its relationship to luminal VT neo-endothelialization, utilizing fluorescence microscopy, histopathology, transmission electron microscopy, and in vivo endothelial cell labeling. We next developed a theranostic IVM fibrin molecular imaging strategy to predict the fibrinolytic response, based on the fibrin accessibility FTP11 imaging signal.

\section{Materials and Methods}

\section{Synthesis of the FTP 11 NIRF fibrin-targeted imaging agent}

FTP11 is a NIRF analog of EP-2104R, a fibrin-targeted MRI agent.(7) Detailed materials and methods describing the synthesis, plasma interference, and biodistribution of FTP11 are available in the online supplement.

\section{Animal Model}

The Institutional Animal Care and Use Committee at Massachusetts General Hospital approved all animal studies. C57/BL6 male mice aged 12 to 16-weeks were anesthetized with ketamine and xylazine $(80 / 12 \mathrm{mg} / \mathrm{kg}$, IP).

\section{Stasis VT}

Ligation-induced stasis VT recapitulates many features of human VT and was induced in the jugular vein.(8-10) A lateral incision followed by blunt dissection exposed the bifurcation of the jugular vein. The external jugular vein was ligated adjacent to the bifurcation using silk suture overlying 6-0 monofilament suture, which was then removed to allow minimal blood flow. Mice were euthanized at either day $4(n=3)$, day $6(n=3)$, day $8(n=3)$, or day $10(n=3)$, perfused with saline, and prepared for histological analysis as below.

\section{Non-stasis VT}

To extend stasis VT findings and to leverage intravital microscopy capabilities, non-stasis VT was induced in the femoral/saphenous vein using chemical injury induced by topical ferric chloride.(11-13) The vein and neurovascular bundle were exposed by blunt dissection through a midline skin incision on the ventral surface of the thigh. A $1 \times 2 \mathrm{~mm}$ strip of \#1 filter soaked in ferric chloride $(7.5 \%)$ for 1 minute was applied to the anterior surface of the vein for 5 minutes. After completion, the area was rinsed with sterile saline and the incision was sutured closed.

\section{Histology and Fluorescence Microscopy}

Following injection of FTP11 and sacrifice, murine veins were perfused with PBS, resected, embedded in OCT and flash frozen. Cryosections of $6 \mu \mathrm{m}$ thickness were obtained. Fluorescence microscopy was performed utilizing an epifluorescence microscope (Eclipse 90i, Nikon) with filters for FTP11-CyAm7 (ex: 673-684nm; em: 765-835nm) and auto-fluorescence (ex: 460-500nm; em: 510-560nm). Adjacent sections were stained with hematoxylin and eosin to assess general morphology. Carstairs' stain identified collagen (blue) and fibrin (red) within thrombosed veins. In vivo labeled CD31 endothelium and neo-endothelium in VT were detected via AF647-targeted filters (ex: 630-670nm; em: $685-735 \mathrm{~nm})$. Histology was obtained from resected venous thrombi ( $\mathrm{n}=3$ per timepoint) without fibrinolysis treatment.

\section{Transmission Electron Microscopy}

For transmission electron microscopy, a subset of venous thrombi ( $n=4$ stasis VT, $n=4$ non-stasis VT) were fixed in Karnovsky's fixative $(2.5 \%$ glutaralydehyde/ $2 \%$ paraformaldehyde) for $24-48 \mathrm{hrs}$ and then rinsed in $0.1 \mathrm{M}$ cacodylate buffer. The specimens were then post-fixed in $2 \% \mathrm{OsO} 4$ in sodium cacodylate buffer and rinsed in $0.1 \mathrm{M}$ cacodylate buffer. Dehydration in a graded series of alcohols followed, and the thrombi were then embedded in Epon 812 and 
polymerized overnight at $60^{\circ} \mathrm{C}$. One micron sections were cut and stained with filtered methylene blue/toluidine blue in borate buffer. Areas of interest were identified and ultrathin sections were cut and collected on uncoated 150 mesh copper grids. The grids were stained with uranyl acetate and lead citrate, and examined on a Philips CM-10 transmission electron microscope. Images were taken using an undermount XR41M cooled CCD camera.

\section{In vivo CD31 staining of thrombus neoendo- thelial cells}

AlexaFluor647-labeled anti-mouse CD31 antibodies (AbD Serotec, MCA2388A647) were injected $(0.5 \mathrm{mg} / \mathrm{kg})$ in the tail vein of mice with day 1 or day 4 non-stasis VT ( $\mathrm{N}=3$ per time point). One hour post-injection, mice were sacrificed and perfused with PBS. Thrombosed tissues and contralateral control tissue (non-thrombosed) were resected, flash-frozen in optimal cutting temperature (OCT) medium, and then underwent fluorescence microscopy (Eclipse 90i, Nikon). A negative control AlexaFluor647-labeled isotype matched antibody (AbDSerotec, MCA1212A647) evaluated in VT demonstrated minimal non-specific binding to thrombus sections (Fig. S3).

\section{Intravital Microscopy (IVM) Imaging of FTP 11 binding to fibrin aging $V T$}

To image fibrin in murine VT in vivo, IVM of FTP11 in non-stasis VT in the femoral/saphenous veins was performed at $1,2,4$ or 6 days post-thrombus induction ( $\mathrm{N}=21$ mice, Fig. $\mathrm{S1})(13)$. Intravenous injection of imaging agents were performed via tail vein catheter. First, the fibrin-specific FTP11 molecular imaging agent $(750 / 767 \mathrm{~nm}$ ex/em, 150 nmoles $/ \mathrm{kg}$ ) was injected intravenously 45 minutes prior to IVM. FITC-modified dextran (FITC-dextran, 490/520nm ex/em, 10mg/kg, MW $2000 \mathrm{kDa}$, Sigma) was injected immediately prior to imaging to enable venous angiography and baseline thrombus area measurement. All mice were serially imaged pre- and 15-, 30-, 45- and 60-minutes post-initiation of fibrinolysis. To avoid inaccurate measurement in thrombus area due to leakage of FITC-dextran into thrombi over the initial one hour IVM session, a second spectrally distinct dextran agent (tetramethylrhodamine (TMR)-modified dextran, 557/576nm ex/em, 10mg/kg, MW $2000 \mathrm{kDa}$ ) was injected prior to the 60 minute imaging timepoint, and used to determine the final thrombus area measurements.(13)

IVM was performed using a custom-built multichannel intravital confocal microscope.(14) Images were collected at 30 frames/second with a field-of-view of $714 \times 714 \mu \mathrm{m}$ with a resolution of 500 $x 500$ pixels. Final images were an average of 15 consecutive frames. For every time point, 3-4 z-stacks covering the entire thrombus length with a z-step of 5 $\mu \mathrm{m}$ were acquired.

Laser lines at $750 \mathrm{~nm}, 491 \mathrm{~nm}$ and $561 \mathrm{~nm}$ were utilized to excite FTP11, FITC-dextran and TMR-dextran, respectively. Fluorescence emissions were collected using corresponding photomultiplier tubes. FTP11 fluorescence was collected using a 770nm longpass filter. FITC-dextran and TMR-dextran fluorescence were collected using a 509-547 $\mathrm{nm}$ and 573-613nm bandpass filter, respectively.(14)

\section{Fibrinolysis of murine VT}

Immediately following pre-fibrinolysis IVM imaging (Fig. S1), mice were administered a bolus of tissue plasminogen activator $(1 \mathrm{mg} / \mathrm{kg}$, Alteplase, Genentech), human plasminogen $(100 \mathrm{mg} / \mathrm{kg}$, Sigma P7999) and heparin (200 U/ kg) via tail vein catheter. As mouse plasminogen resists activation by human tPA,(15) exogenous human plasminogen was administered to augment plasmin generation and fibrinolysis. After the bolus injections, mice received a continuous infusion of tPA $(9 \mathrm{mg} / \mathrm{kg} / \mathrm{hr})$ and heparin $(50$ $\mathrm{U} / \mathrm{kg} / \mathrm{hr}$ ) over the entire imaging session utilizing a syringe pump. Heparin and tPA doses were based on previous work in various thrombosis models.(16-21) For each experiment, the tissue plasminogen activator amidolytic activity was verified using an established commercial chromogenic substrate S-2288.(16)

\section{Image Analysis}

Fusion of multiple z-stacks along the $x_{-}, y$ - and $\mathrm{z}$-dimensions generated whole thrombus datasets (NIH ImageJ version 1.44o, Bethesda, MD).(13) All image analyses were conducted on mid-lumen coronal cross-sectional areas of $40 \mu \mathrm{m}$ in thickness that was centered at $40 \mu \mathrm{m}$ below the vein wall-lumen interface. Each dataset was thus comprised of eight contiguous z-images averaged to make a coronal stack for analysis. For each time point, a thrombus region-of-interest was defined in the FITC-dextran channel and used for analysis of the FTP11 channel.

The net lysis at a given timepoint was defined as (Thrombus areapre-lysis -Thrombus area $\left._{\text {timepoint }}\right) /\left(\right.$ Thrombus area $\left.a_{\text {prelysis }}\right) * 100 \%$. The FTP11 fluorescence signal was quantified as the integrated signal density (ISD, thrombus area * mean signal intensity in the thrombus). The normalized change in the FTP11 signal was calculated (FTP11 ISD $_{\text {pre-lysis }}-F T P 11$ ISD $\left._{\text {timepoint }}\right) /\left(\right.$ FTP11 ISD pre-lysis $_{\text {) T }}$. The FTP11 target-to-background ratio (TBR) was calculated as the mean FTP11 signal intensity within the 
thrombus ROI divided by mean FTP11 signal intensity in the non-thrombosed venous lumen. Segmental analysis was accomplished using line-by-line analysis of the FITC-dextran and FTP11 channels at the initial and final time points. Data was then normalized between thrombi by calculating changes in area or FTP11 signal as a percent of total thrombus length. The pooled proximal 5\% and distal 5\% ends of the thrombus were then compared to the $90 \%$ interior segment of thrombus.

\section{Statistical Analysis}

All statistical analyses were completed using Prism software version 5.0c (GraphPad). Analyses between two or more groups were performed using nonparametric Kruskal-Wallis test with post-test Dunn's multiple comparison test between two groups. Correlation was quantified using linear regression and the Spearman coefficient was calculated. Data is presented as mean \pm standard deviation.

\section{Results}

\section{FTP 11 -fibrin specific accumulation in murine venous thrombi diminishes with age}

Fluorescence microscopy of VT sections demonstrated that FTP11 binding diminished as a function of thrombus age in both stasis and non-stasis VT (Fig. 1 and Fig. S2, respectively). In earlier stage VT, FTP11 bound to the luminal surface of venous thrombi. Although FTP11 binding was limited to the luminal surface, fibrin was present in venous thrombi at all time-points (Carstairs' stain, red stain, Fig. 1 center column). Histopathological analysis of stasis VT revealed increasing venous thrombus cellularity, and decreasing fibrin content, as thrombi aged. Similar results were observed in non-stasis VT, however the time course of reduced FTP11 fibrin binding was accelerated (Fig. S2).

\section{Development of a luminal neoendothelial bar- rier between fibrin and the circulating blood}

The data above revealed that fibrin accessibility to the blood-based fibrin-targeted FTP11 agent declined as VT aged. As neo-endothelialization occurs as part of VT resolution(5) we investigated whether a developing luminal neoendothelial cell layer might limit contact of the circulating blood with fibrin within VT, and provide insight into diminished FTP11 binding to fibrin over time. To examine the thrombus-lumen interface at ultrahigh resolution, transmission electron microscopy (TEM) of VT sections were performed in day 2 and day 10 stasis-induced VT (Fig. 2A). TEM revealed the sub-cellular structure of the venous thrombus and thrombus-lumen interface. Both day 2 and day 10 venous thrombi were com- posed of fibrin (Fig. 2A, dark fibers on TEM, green arrowheads). In early day $2 \mathrm{VT}$, superficial fibrin within thrombi directly contacted red blood cells from the circulating blood (Fig. 2A, left). In contradistinction, day 10 VT exhibited a neoendothelial layer that encased the luminal surface of venous thrombi, and separated fibrin from the circulating blood (Fig. 2A, right). These findings occurred similarly in non-stasis DVT (Fig. 2B and Fig. S3).

Next, to further determine whether the developing interface in aging VT represented a neo-endothelial layer, mice with non-stasis VT at day 1 or day 4 were intravenously injected with a fluorescently labeled CD31-specific antibody to demarcate endothelial cells. Fluorescence microscopy of VT sections revealed that CD31-positive endothelial cells coated the luminal thrombus surface of subacute day $4 \mathrm{VT}$, in contrast to younger day $1 \mathrm{VT}$ that exhibited scant CD31 signal (Fig. 3). Contralateral non-thrombosed control femoral veins showed circumferential CD31 expression indicating a normal endothelial layer (Fig. S3A). A fluorescently labeled negative isotype control antibody did not bind day 1 or day 4 VT or non-thrombosed vein sections (Fig. S4B).

\section{Assessment of fibrin accessibility in vivo via intravital microscopy of FTP11 deposition}

For in vivo investigation of fibrin accessibility, non-stasis VT in the femoral/saphenous veins was utilized due to its relative immobility and its size, which facilitate high-resolution intravital microscopy (IVM) of murine VT.(11-13). To image fibrin, the fibrin-specific in vivo NIRF fluorescence molecular imaging agent FTP11(6) was intravenously injected in mice bearing VT of different ages. IVM revealed that fibrin-targeted FTP11 bound to the thrombus luminal surface of day 1 and day 2 thrombi (Fig.4), in a pattern consistent with fluorescence microscopy data above (Fig. S2). In contrast, FTP11 binding declined with thrombus age (Fig. 5, FTP11 target-to-background ratio (TBR) day 1: $1.88 \pm 0.46$; day 2: $1.32 \pm 0.30$, day 4 : $0.98 \pm 0.15$, and day $6: 1.06 \pm 0.14, p=0.002$ ). These findings further suggest that a developing neo-endothelial barrier may prevent agents in the circulating blood such as FTP11 from accessing fibrin in vivo.

\section{Fibrinolytic efficacy of VT diminishes with thrombus age}

The circulating blood contains key components of the fibrinolytic system including plasminogen and endogenous and exogenous plasminogen activators. If the development of a luminal neoendothelial layer can prevent blood components from accessing fibrin within the thrombus, as demonstrated above, we hy- 
pothesized that fibrinolysis would be impaired in aging thrombus, coincident with luminal neo-endothelialization. To test this hypothesis in vivo, serial IVM was performed in $\mathrm{N}=21$ mice with non-stasis VT treated with a one-hour intravenous tPA plus heparin protocol (Fig. S1). Fluorescent-dextran venography enabled visualization and reliable IVM quantification of venous thrombi area (13) both pre- and post-fibrinolysis (Fig. 5A-C). The net VT fibrinolysis after 60 minutes of tPA infusion was significantly higher in earlier-stage venous thrombi (day 1, 27 $\pm 13 \%$; day 2, 25 $\pm 16 \%$ ) compared to older VT (day 4, 5.9 $\pm 4.2 \%$; day 6, $5.3 \pm 3.8 \%$; $p=0.002$, Fig. 5D). Analysis of serial IVM datasets every 15 minutes revealed that the rate of fibrinolysis and the rate of reduction in FTP11 signal (fibrin dissolution) were also higher in day 1 and day 2 thrombi (Fig. S5A and S5B, $\mathrm{p}<0.05)$.
FTP11

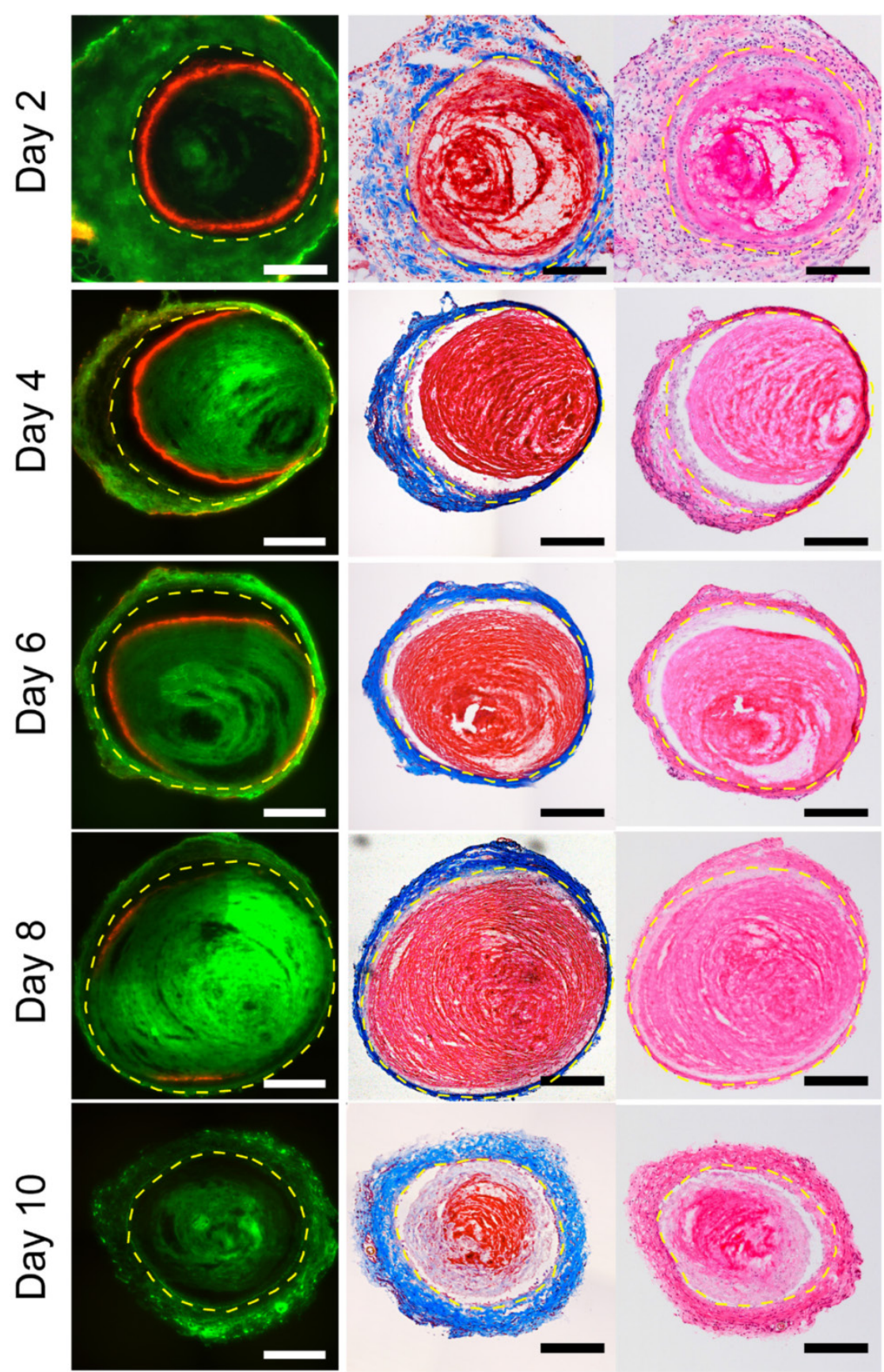

Figure 1. Histological assessment of FTP11 binding to stasis-induced venous thrombosis of increasing age. Fluorescence microscopy of FTP11 accumulation, Carstairs' and hematoxylin and eosin (H\&E) staining were analyzed across various aged thrombi (left, middle and right columns, respectively). Fluorescence from FTP11 (red) was localized to the luminal edge of the acute thrombi. FTP1 1 accumulation decreased with thrombus age. Carstairs' staining demonstrated fibrin-rich thrombi (red) and collagen fibers in the vein wall (blue). H\&E staining showed increasing cellularity throughout the thrombi. Autofluorescence is shown in green. Scale bar, $200 \mu \mathrm{m}$. 


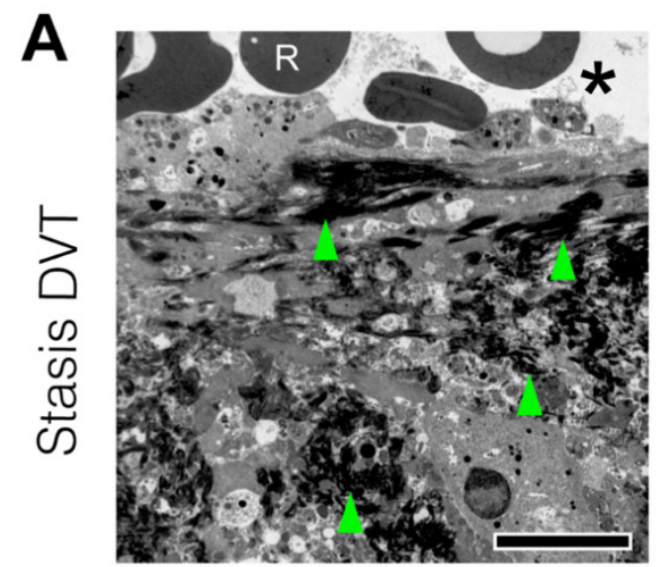

Early Day 2

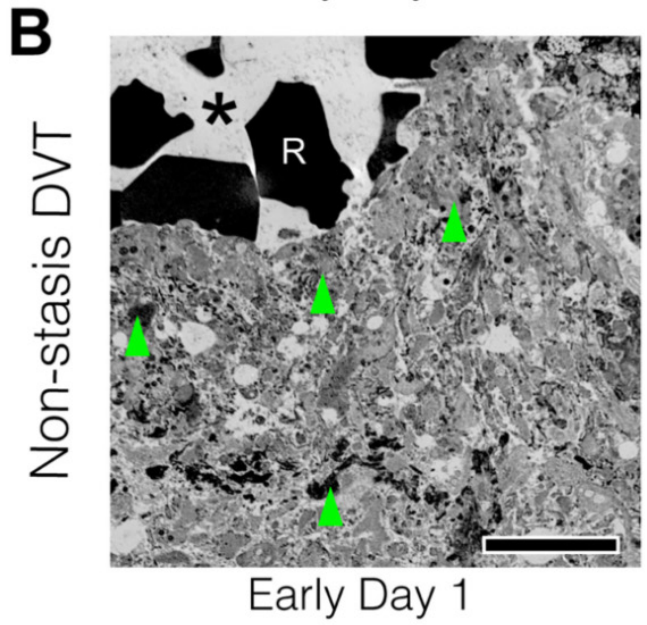

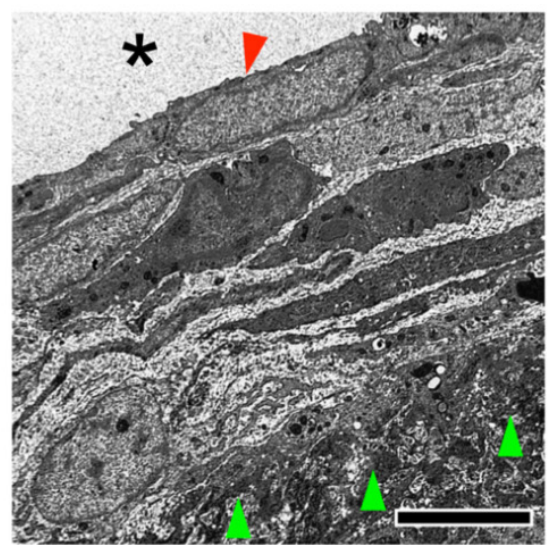

Subacute Day 10

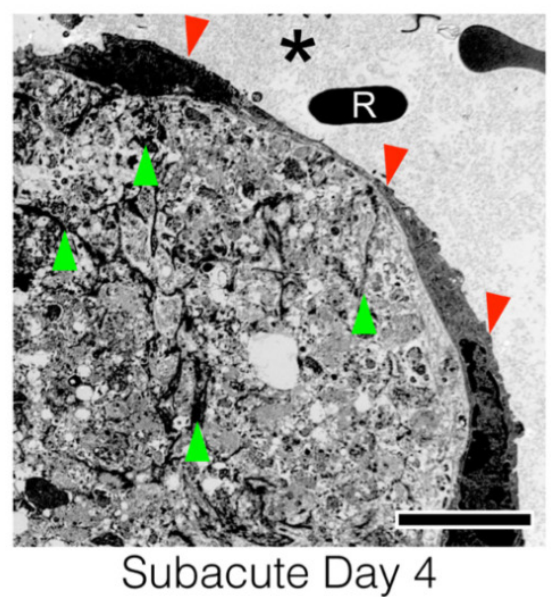

Figure 2. Transmission electron microscopy of acute and subacute stasis and non-stasis venous thrombosis. Representative acute and subacute venous thrombi-lumen interfaces are shown for both (A) stasis (B) non-stasis venous thrombi. Micrographs from acute VT in direct contact with luminal RBCs. Fibrin (green arrows) can be seen throughout the thrombi at both timepoints. Subacute VT exhibit a luminal neoendothelial cell layer (red arrowhead) that encases the thrombi. Scale bar, $5 \mu m$. Asterisk indicates lumen. $R=$ red blood cell.

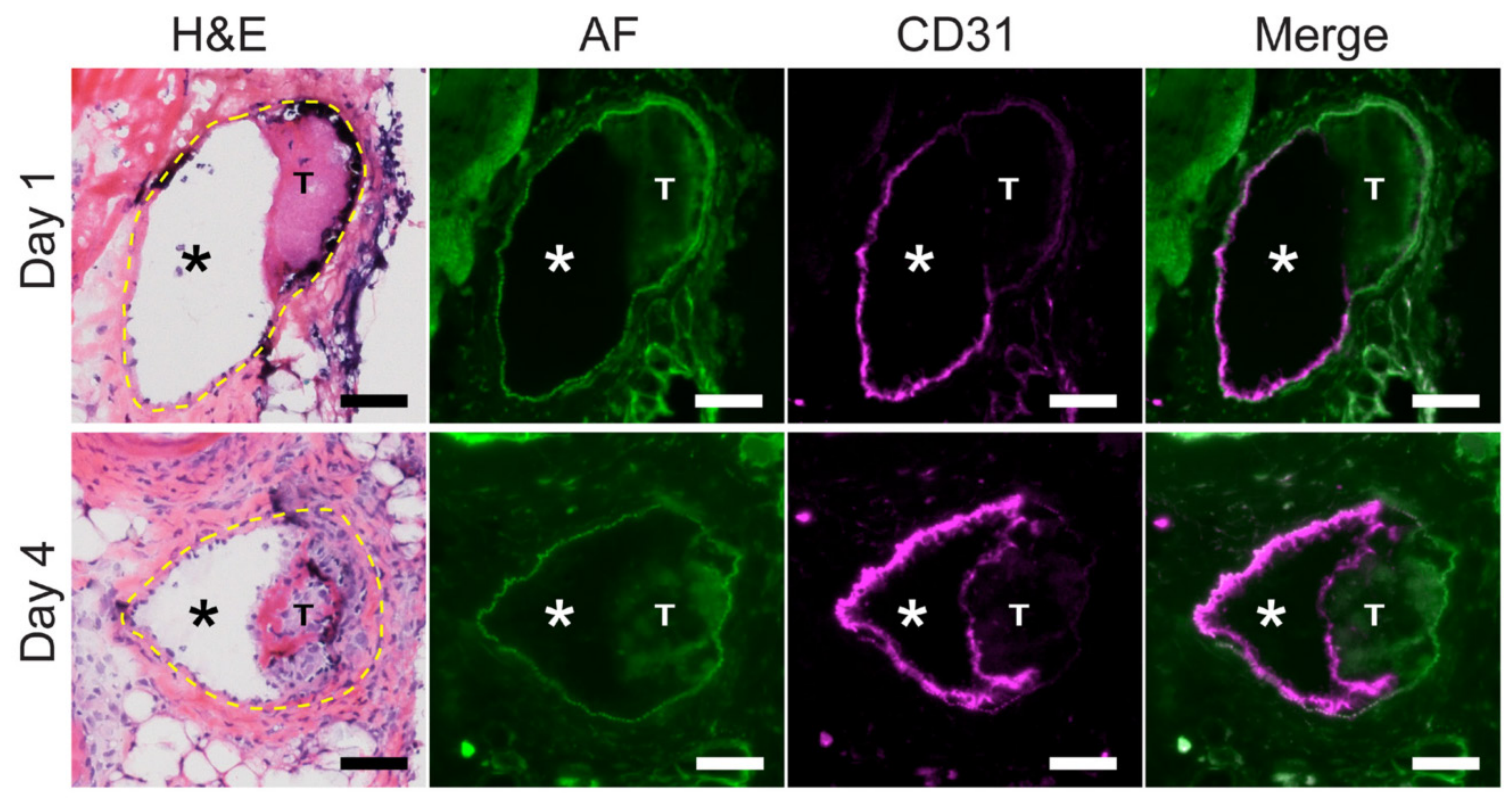

Figure 3. Venous thrombus age and the development of a CD31-positive luminal neoendothelial cell barrier layer. Fluorescence microscopy of VT sections was performed on mice after an intravenous injection of a fluorescent anti-mouse CD31 antibody. In a representative day 1 VT, minimal CD31 signal (magenta pseudocolor) overlies the thrombus, while in contrast a representative day $4 \mathrm{VT}$ shows a thrombus-encased surface of CD31-positive neoendothelial signal at the luminal surface. H\&E sections (dashed yellow line) and autofluorescence (AF, green pseudocolor) delineate the vein, as well as the lumen $\left.{ }^{*}\right)$ and thrombosis (T). CD31-positive endothelial cells line the non-thrombosed venous lumen in both day 1 and day 4 animals. Scale bar, $50 \mu \mathrm{m}$. 


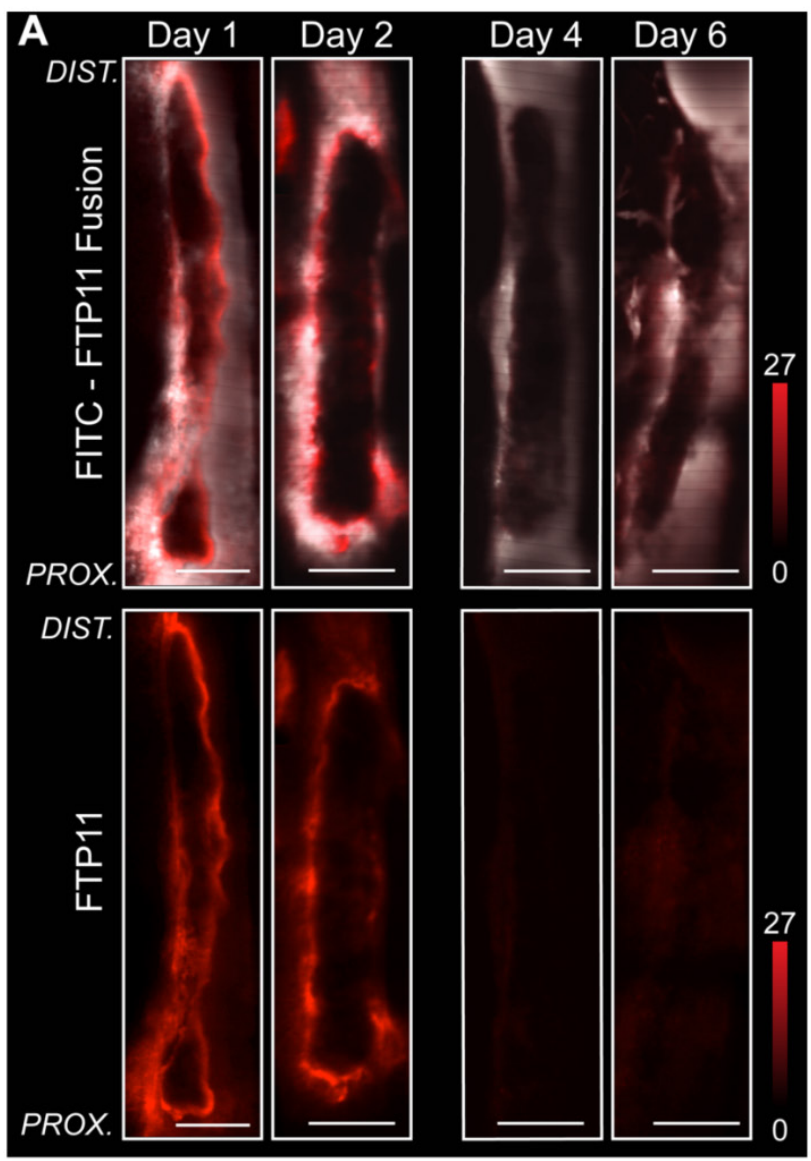

B

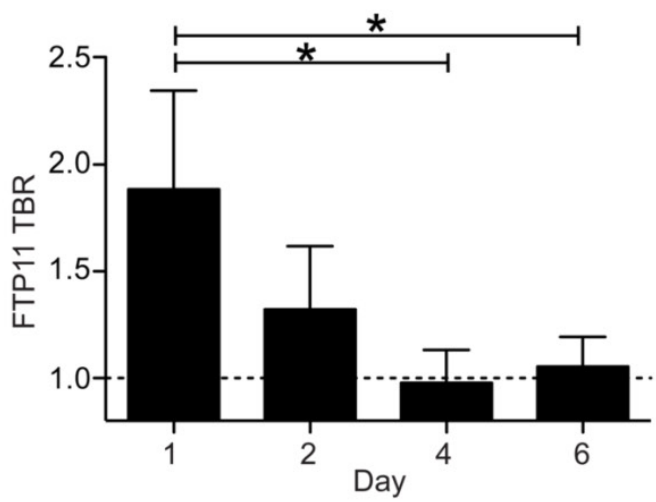

Figure 4. Assessment of fibrin accessibility in vivo as a function of venous thrombus age. Murine femoral/saphenous non-stasis VT were created to enable high-resolution intravital microscopy. (A) Intravital microscopy venographs, using FITC-dextran as a blood pool agent, show VT as hypointense regions in the lumen of the vein. FTP11 (red) binds fibrin primarily around the rim of the acute (day 1 and 2) VT, but exhibits minimal binding to subacute day 4 and $6 \mathrm{VT}$. The proximal vessel is at the bottom of each image. (B) Quantification of the FTP11 fibrin target-to-background ratios (TBR) reveal significantly higher FTPI 1 accumulation in day $1 \mathrm{VT}$ compared to day 4 and day 6. Scale bar, $200 \mu \mathrm{m}$. ${ }^{*} \mathrm{p}<0.05$

Additional experiments explored the effects of murine plasma on FTP11 binding to fibrin, and the biodistribution of FTP11. FTP11 bound fibrin in vitro without significant interference from murine plasma proteins (Fig. S7). These results are consistent with the finding that FTP11 showed good fibrin target-to-background ratios in acute VT. Consistent with FTP11's short blood half-life of 2.82 minutes (6) and low molecular weight, FTP11 biodistribution studies revealed rapid clearance through the urinary system (Fig. S8).

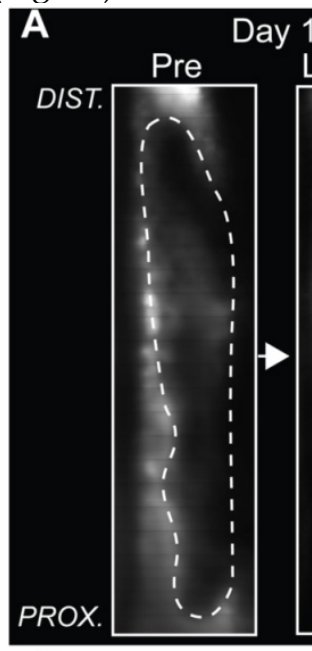

C

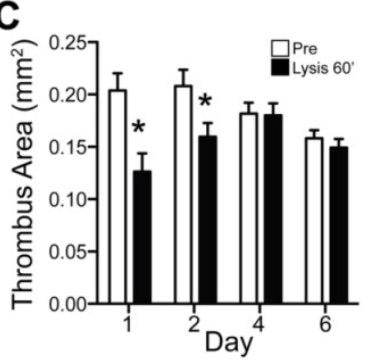

B Day 4
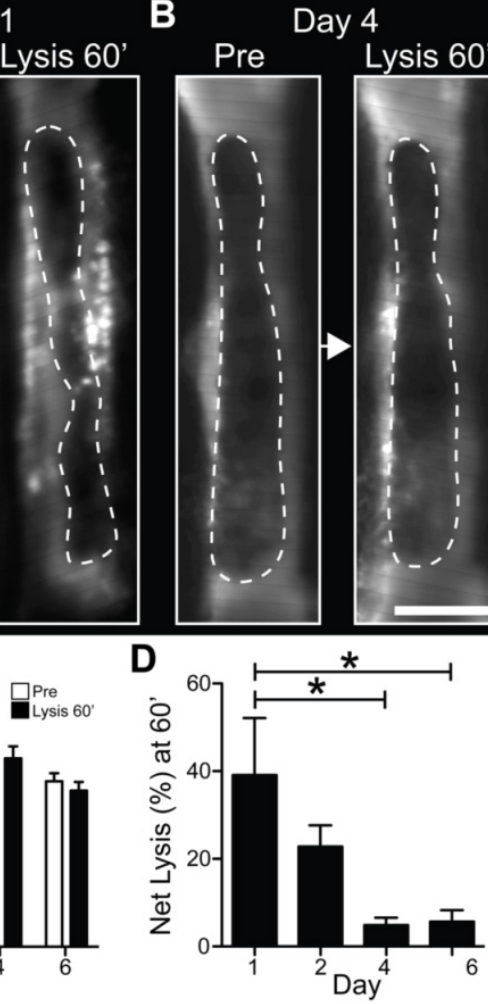

Figure 5. Fibrinolysis diminishes as a function of venous thrombus age. Mice with VT received 1 hour of intravenous tissue plasminogen activator infusion. Representative IVM dataset of pre-lysis and 60 minute post-lysis VT IVM images in (A) day 1 and (B) day 4 VT. The baseline pre-lysis VT area is demarcated by the blood pool imaging agent FITC-dextran (dotted lines outline the dark thrombus within the bright FITC-dextran luminal signal). Representative IVM data reveal that younger day 1 VT experience greater fibrinolysis compared to subacute day 4 VT (Lysis 60' images). (C,D) Quantification of net fibrinolysis from the pre-lysis and 60 minute lysis IVM images show significantly greater fibrinolysis in acute day 1 VT compared to day 4 and 6 VT. $* \mathrm{p}<0.05$.

\section{The in vivo FTP 11 fibrin accessibility signal predicts the efficacy of exogenous venous thrombosis fibrinolysis}

As the FTP11 fibrin molecular imaging signal identified blood-accessible fibrin in VT (Fig. 1, Fig. 4 and Fig. S2), we hypothesized that the baseline FTP11 fibrin signal would identify VT amenable to fibrinolysis, and thus offer a theranostic strategy to predict the efficacy of exogenous tPA-triggered fibrinolysis. In earlier stage day 1 and day 2 VT, the thrombus and FTP11-positive areas demonstrated substantial fibrinolysis during the one hour tPA protocol (Fig. 6A). In contrast, subacute day 4 and day 6 thrombi experienced weaker thrombus and FTP11 signal reductions during lysis. The changes in the FTP11 signal, or in vivo fibrin dissolution, correlated well with the net thrombolysis obtained from IVM pre- and post-venography $(\triangle \mathrm{FTP} 11$ integrated signal density vs. net fibrinolysis (\%) at 60 minutes, $r=0.633, p=0.001$, 
Fig. 6C). Furthermore, the baseline FTP11 fibrin accessibility signal predicted the net fibrinolysis achieved after 60 minutes of tPA infusion $(r=0.642$, $\mathrm{p}=0.001$, Fig. $6 \mathrm{D}$ ). These findings demonstrate that FTP11 identifies blood-accessible fibrin that can undergo fibrinolysis, and that the FTP11 fibrin accessibility signal can predict the efficacy of exogenous fibrinolysis.

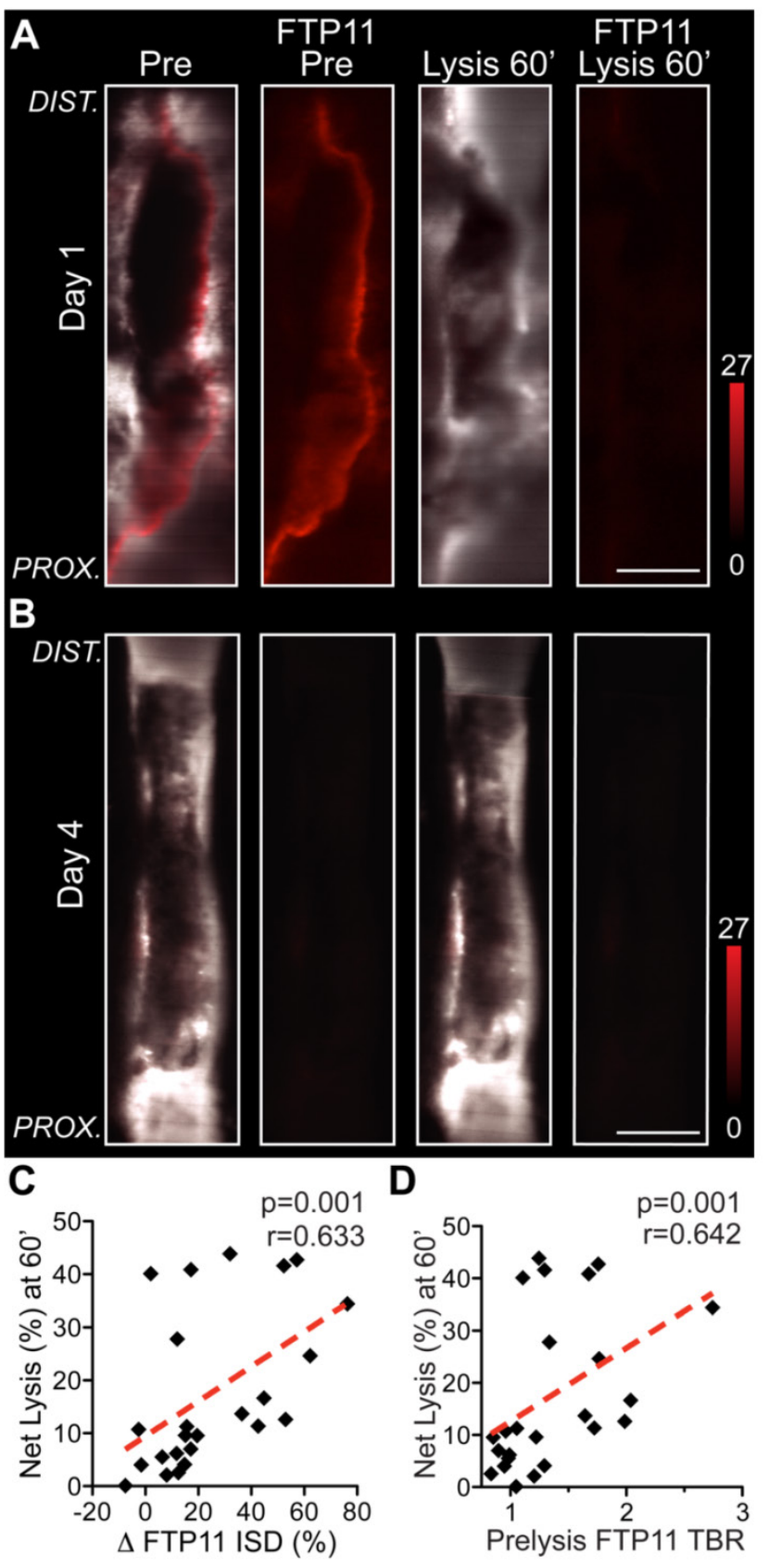

Figure 6. In vivo baseline FTP11 fibrin accessibility signal in VT, and the subsequent response to fibrinolysis. Representative IVM micrographs of FTP11 binding pre- and post-lysis from (A) acute day 1 VT show FTPII fibrin signal dissolution following 60 minutes of systemic tPA-induced fibrinolysis. The first and third columns show a fusion of the FITC-dextran angiogram (greyscale) and the FTP11 fibrin signal (red). The second and fourth columns show the FTP11 fibrin NIRF signal (red) by itself, equally windowed across the images. (B) Subacute day 4 VT exhibit little FTPII signal at baseline, and experience little fibrinolysis. (C) The \%FTP1 1 signal loss ( $\triangle I S D)$ during lysis tracked well with the net lysis achieved at 60 minutes $(r=0.633, p=0.001)$. (D) The pre-lysis baseline FTPI1 signal predicted the net total fibrinolysis at 60 minutes $(r=0.642, p=0.001)$

\section{The proximal and distal edges of venous thrombi experience greater fibrinolysis}

As the edges of thrombi have greater surface areas exposure to blood, we hypothesized that the fibrinolysis would be greater at the edges compared to the thrombus interior. Segmental IVM line-by-line analysis (13) of day 1 mice undergoing FTP11-injection and the fibrinolysis protocol revealed that thrombolysis was significantly greater at the pooled proximal and distal edges (Fig. 7A and 7B, edges: $55.5 \pm 24.1 \%$ vs. the interior: $23.2 \pm 21.9 \%$, $\mathrm{p}=0.001$; edges were defined as the combined outer $10 \%$ of each thrombi). Moreover, thrombus edges experienced significantly greater FTP11-labeled fibrin dissolution ( $\triangle$ FTP11 integrated signal density) compared to the thrombus interior (Fig. 7C and 7D, edges: $72.3 \pm 22.1 \%$ vs. the interior: $42.5 \pm 36.2 \%, p=0.004)$.
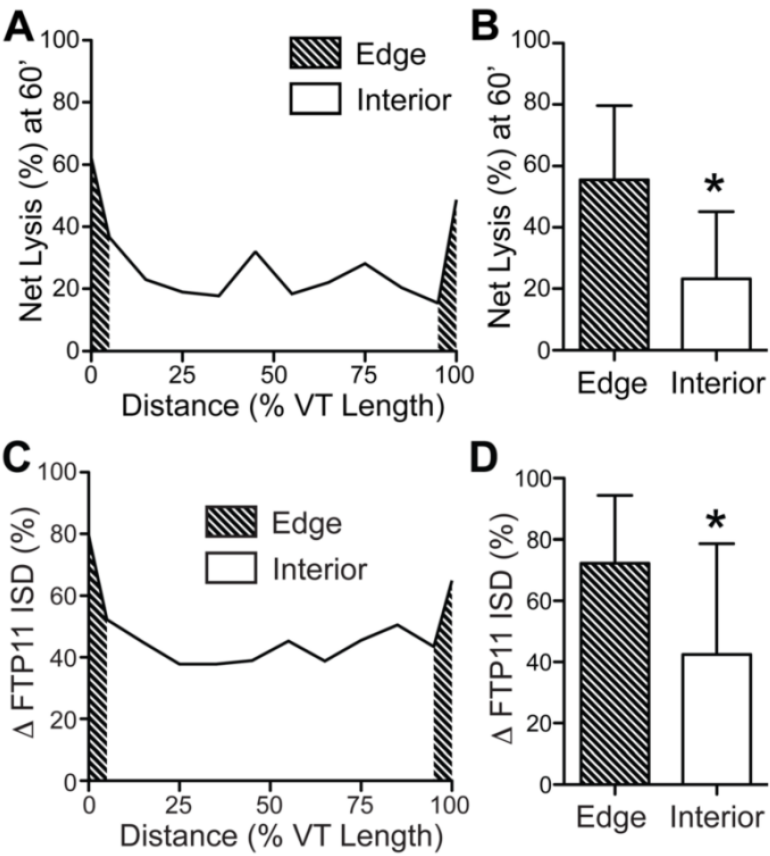

Figure 7. Augmented fibrinolysis occurs at the thrombus edges, as compared to the thrombus interior, in day 1 VT. (A, B) Line-by-line IVM image analysis of the net fibrinolysis shows a significant increase in fibrinolysis at the proximal and distal edges (shaded area), as compared to the thrombus interior. (C, D) Line-by-line IVM image analysis also demonstrates significantly greater FTP11 signal reduction (change in FTP11 ISD) occurs at the thrombus edges (shaded area), as compared to the thrombus interior. The proximal and distal edges were pooled for the graphs in (B) and (D). ISD, integrated signal density. ${ }^{*} \mathrm{p}<0.05$.

\section{Discussion}

The results of this experimental molecular-structural theranostic study demonstrate that the fibrinolytic efficacy of murine VT diminishes over time, and is linked to reduced blood accessibility to fibrin that coincides with the development of a shielding luminal neoendothelial barrier layer. These results have implications for optimizing the use of 
clinical fibrinolytic therapy for VTE dissolution and reperfusion, including systemic fibrinolytic agents and catheter-directed therapy.

Fibrin is the major proteinaceous component of the initial thrombus scaffold and provides a surface for thrombus propagation and eventual vessel occlusion. Fibrinolytic therapy with tPA drives the conversion of plasminogen to plasmin. Plasmin then cleaves chains of fibrin to accelerate thrombus dissolution.(22) In vitro studies of fibrinolysis demonstrate that fibrin network pore size, fiber density, fibrin thickness, and RBC composition partially control of the rate of clot lysis by controlling enzyme concentrations and accessibility. $(23,24)$ Additionally, fibrinolysis studies have showed tPA binding primarily on the surface of the thrombus to a depth of 5-8 $\mu \mathrm{m}$, indicating a role for thrombus permeability and organization in fibrinolysis. $(25,26)$ However, in vitro models do not recapitulate the cellular processes, including endothelialization, and the fluid mechanics that influence in vivo fibrin accessibility and fibrinolysis of venous thrombi. This point is particularly germane to venous thrombi that incorporate several cellular components including neutrophils, macrophages, and endothelial cells during acute formation and resolution.

Therefore, to investigate the role of fibrin accessibility in fibrinolysis in vivo, we utilized an established fibrin-specific NIRF molecular imaging agent FTP11.(6) FTP11 is a peptide derived from phage display that selectively binds to fibrin with little interference from fibrinogen or albumin (7), or murine plasma (Fig. S7). The key observation of this study is that in both stasis and non-stasis VT, FTP11 distributed along the luminal thrombus surface, but exhibited diminished binding with thrombus age.

A mechanism that contributed to reduced fibrin FTP11 binding was revealed by high-resolution transmission electron microscopy (TEM) and in vivo CD31 labeling of neoendothelial cells. These results demonstrated that luminal neo-endothelialization encased subacute VT, and hindered circulating blood and FTP11 from interfacing with the underlying thrombus and fibrin, respectively (Fig. 2 and 3). This neoendothelial layer was absent in early VT In both models. Thereafter, serial IVM of thrombus area and structure revealed that the degree of baseline FTP11 accumulation, or the fibrin accessibility signal, predicted the extent of tPA-induced fibrinolysis. Both the baseline FTP11 signal and the magnitude of fibrinolysis were higher at the proximal and distal thrombus edges, as compared to the thrombus interior, indicating a surface area-dependent process. The overall FTP11 IVM, fluorescence microscopy, histology, TEM, and in vivo fibrinolysis results provide evidence that:
(1) VT fibrinolysis diminishes with thrombus age; (2) VT fibrinolysis relates to the accessibility of fibrin to blood-based fibrinolytic enzymes, which is reduced as a neoendothelial layer forms a barrier around an aging venous thrombus; and (3) the in vivo FTP11 fibrin accessibility signal predicts the efficacy of exogenous fibrinolysis.

Thrombus neovascularization is a critical aspect of VT resolution and restoring blood flow. These processes serve to limit thrombus propagation and facilitate thrombus organization.(5) As resolving venous thrombi have been shown to have increased venous blood flow over time due to thrombus neovascularization(27), the reduction in FTP11 binding to aging VT is not likely due to reduced venous blood flow. The current study results reveal that a luminal neo-endothelial layer can coat aging thrombi, preventing access of blood components to fibrin (e.g. FTP11, Fig. 4), and thus providing insight into how VT fibrinolysis diminishes with age. In this study both a stasis and chemical injury model of VT were utilized. In both models, VT form a single endothelial layer on the surface of a fibrin-rich thrombus. The main difference between these models is the time course of neoendothelialization, as demonstrated in Figs. 2 and 3. In the non-stasis model, thrombi form immediately and there is an increased inflammatory response due to the chemical injury of surrounding tissue. In the stasis model, thrombus formation is a more gradual process and neoendothelialization is delayed until day 8 .

A recent study by Andia et al. employed an MRI analogue of FTP11, termed EP-2104R, to investigate fibrinolysis using experimental murine VT generated by stasis and mechanical endothelial disruption.(28) This study and our study are consistent in that the intensity of FTP11 or EP-2104R signal predicted the response to experimental fibrinolysis in vivo. By demonstrating that the FTP11 signal reflects the blood accessibility to fibrin, as related to the absence of an age-dependent shielding neoendothelial layer, our experimental results extend the study by Andia et al.(28), and suggest that in addition to fibrin maturation, the neoendothelial layer may play a significant role in mediating fibrinolytic resistance of venous thrombi. In addition, the recognition that a neoendothelial layer could limit fibrin-targeted imaging agent binding could help explain the unexpectedly low sensitivity of EP2104-R for detecting clinical venous thrombi $(29 \%$ of venous thrombi, with only 1 new thrombus identified by EP2104-R beyond baseline non-contrast MRI).(29) It may be plausible that, similar to FTP11 in this study, EP2104-R also binds younger thrombi devoid of neoendothelium, and thus with blood-accessible fibrin. 
At present, clinical fibrinolysis of deep vein thrombosis (DVT) is recommended for relatively recently formed thrombi, typically less than 14 days old. $(3,4)$ However, current clinical assessment of VT age is based on patient history, which may be unreliable. For example, as venous thrombi can form insidiously within the vein valve pocket sinuses,(30) clinical VT might be many days old before causing symptoms. Therefore reliance on clinical history alone could significantly underestimate the age of a DVT, and thereby overestimate its responsiveness to fibrinolysis. As fibrinolytic therapy confers a significant, potentially life-threatening hemorrhage risk, molecular imaging of fibrin could thus potentially improve the benefit-to-risk ratio of fibrinolytic therapy, by specifically identifying DVT or PE with blood-accessible fibrin and more likely to respond to fibrinolysis. Based on the data from the current study and previous EP2104-R imaging studies, clinical molecular imaging of fibrin signal intensity in VTE could provide a measure of thrombus age, preferentially identify younger thrombi, and ultimately provide a novel approach to identify patients more likely to respond to fibrinolytic therapy. EP2104-R is the MRI analogue of FTP11 that has already been tested in phase II clinical trials of VTE imaging.(29) MRI allows noninvasive visualization of deep tissue, while confocal NIRF imaging requires exposure of the vessel due limited light penetration. Therefore noninvasive MRI is more likely to be a clinical fibrin molecular imaging platform. In this experimental study, however, intravital NIRF microscopy provided the high-resolution, real-time, dynamic imaging of fibrin binding and dissipation (Fig. 4, 5; Fig. S5, Fig. S6) that was necessary to understand the effects of thrombus age on FTP11 binding. In the future, clinical NIRF imaging of fibrin deposition might be enabled via small NIRF catheters that can image many vascular beds. $(31,32)$

Limitations of this study are present. Stasis VT requires larger vein sizes (jugular, inferior vena cava) that are subject to greater motion artifact and greater undersampling by depth-limited confocal IVM; therefore we selected non-stasis VT in the smaller femoral/saphenous vein to provide a reproducible, nearly motion-free VT area for high-resolution IVM and for reliably quantification of in vivo thrombus area.(13) In addition, it is possible that alternative DVT models might alter the time course of fibrin generation(28) and neoendothelialization of thrombi. Other factors may also influence the observed thrombus age-dependent reductions in fibrinolysis and FTP11 binding and dissolution rates, such as fibrin structure (fiber thickness, density, cross-linked status), red blood cell density of the thrombus,(24) and differential expression of fibrinolytic inhibitors.(33) Due to the limited light penetration of confocal IVM to $100-150 \mu \mathrm{m}$, our analyses were performed on 2D slices over a thickness of $40 \mu \mathrm{m},(13)$ rather than true full volume measurements. In future studies, mechanistic approaches that alter the rate of VT endothelialization will provide greater understanding of the role of neo-endothelialization in fibrinolytic resistance.

In conclusion, aging experimental venous thrombi demonstrate reduced accessibility of fibrin to blood and reduced fibrinolysis, coincident with the development of a luminal thrombus neoendothelial layer that separates VT fibrin from the blood. FTP11-based fibrin molecular imaging illuminates fibrin accessibility in vivo, and predicts fibrinolytic responsiveness in vivo. These findings provide new insights into mechanisms underlying VT fibrinolytic resistance, and could be useful in optimizing the timing of fibrinolytic therapies for venous thrombi and pulmonary embolism.

\section{Significance}

This experimental fibrin molecular-structural imaging and ultrastructural study provides new insights into in vivo mechanisms governing the fibrinolytic response of murine venous thrombi. Intravital microscopy of VT demonstrated that the fibrinolytic response diminishes with increasing thrombus age. These temporal reductions occur coincident with the development of a thrombus-encompassing neoendothelial barrier layer that limits contact of blood components to fibrin within VT. Fibrin-based molecular imaging of VT further predicted the response to tissue plasminogen activator therapies. This study methodology further provides a platform anticipated to be useful in investigating novel pharmacological and genetic modulators of fibrinolysis. Finally, clinical molecular imaging of fibrin could potentially identify VTE more likely to respond to fibrinolytic therapy.

\section{Supplementary Material}

Figures S1-S8. http://www.thno.org/v05p1317s1.pdf

\section{Abbreviations}

AF: autofluorescence; DVT: deep vein thrombosis; FITC: fluorescein isothiocyanate; FM: fluorescence microscopy; H\&E: hematoxylin and eosin; IVM: intravital microscopy; NIRF: near infrared fluorescence; TEM: transmission electron microscopy; TBR: target-to-background ratio; tPA: tissue plasminogen activator; VT: Venous thrombosis; VTE: Venous thromboembolism 


\section{Acknowledgements}

The authors thank Margaret Sherwood and Megan Scanlan for assistance and expertise in TEM. This research was supported in part by the American Heart Association (\#12PRE11160000, A.F.S.; \#13POST14640021, T.H.; \#13GRNT17060040, F.A.J.) and US National Institutes of Health (R01 HL108229 and R01 HL122388-01A1, F.A.J.; TPEN HHSN268201000044C and R01 HL122238, J.R.M; T32 HL076136, C.W.K.; R01 HL92750, G.R.).

\section{Competing Interests}

The authors have declared that no competing interest exists.

\section{References}

1. Enden T, Haig Y, Klow NE, Slagsvold CE, Sandvik L, Ghanima W, et al. Long-term outcome after additional catheter-directed thrombolysis versus standard treatment for acute iliofemoral deep vein thrombosis (the CaVenT study): a randomised controlled trial. Lancet. 2012;379(9810):31-8.

2. Meyer G, Vicaut E, Danays T, Agnelli G, Becattini C, Beyer-Westendorf J, et al. Fibrinolysis for patients with intermediate-risk pulmonary embolism. N Engl J Med. 2014;370(15):1402-11.

3. Kearon C, Akl EA, Comerota AJ, Prandoni P, Bounameaux H, Goldhaber SZ, et al. Antithrombotic therapy for VTE disease: Antithrombotic Therapy and Prevention of Thrombosis, 9th ed: American College of Chest Physicians Evidence-Based Clinical Practice Guidelines. Chest. 2012;141(2 Suppl):e419S-94S.

4. Meissner MH, Gloviczki P, Comerota AJ, Dalsing MC, Eklof BG, Gillespie DL, et al. Early thrombus removal strategies for acute deep venous thrombosis: clinical practice guidelines of the Society for Vascular Surgery and the American Venous Forum. J Vasc Surg. 2012;55(5):1449-62.

5. Modarai B, Burnand KG, Humphries J, Waltham M, Smith A. The role of neovascularisation in the resolution of venous thrombus. Thromb Haemost. 2005;93(5):801-9.

6. Hara T, Bhayana B, Thompson B, Kessinger CW, Khatri A, McCarthy JR, et al. Molecular imaging of fibrin deposition in deep vein thrombosis using fibrin-targeted near-infrared fluorescence. JACC Cardiovasc Imaging. 2012;5(6):607-15

7. Overoye-Chan K, Koerner S, Looby RJ, Kolodziej AF, Zech SG, Deng Q, et al. EP-2104R: a fibrin-specific gadolinium-Based MRI contrast agent for detection of thrombus. J Am Chem Soc. 2008;130(18):6025-39.

8. Diaz JA, Obi AT, Myers DD, Jr., Wrobleski SK, Henke PK, Mackman N, et al. Critical review of mouse models of venous thrombosis. Arterioscler Thromb Vasc Biol. 2012;32(3):556-62.

9. Hara T, Truelove J, Tawakol A, Wojtkiewicz GR, Hucker WJ, MacNabb MH, et al. 18F-fluorodeoxyglucose positron emission tomography/computed tomography enables the detection of recurrent same-site deep vein thrombosis by illuminating recently formed, neutrophil-rich thrombus. Circulation. 2014:130(13):1044-52.

10. Kessinger CW, Kim JW, Henke PK, Thompson B, McCarthy JR, Hara T, et al. Statins Improve the Resolution of Established Murine Venous Thrombosis: Reductions in Thrombus Burden and Vein Wall Scarring. PLoS ONE. 2015;10(2):e0116621.

11. Jaffer FA, Tung $\mathrm{CH}$, Gerszten RE, Weissleder R. In vivo imaging of thrombin activity in experimental thrombi with thrombin-sensitive near-infrared molecular probe. Arterioscler Thromb Vasc Biol. 2002;22(11):1929-35.

12. Jaffer FA, Tung CH, Wykrzykowska JJ, Ho NH, Houng AK, Reed GL, et al. Molecular imaging of factor XIIIa activity in thrombosis using a novel, near-infrared fluorescent contrast agent that covalently links to thrombi. Circulation. 2004;110(2):170-6.

13. Ripplinger CM, Kessinger CW, Li C, Kim JW, McCarthy JR, Weissleder R, et al. Inflammation modulates murine venous thrombosis resolution in vivo: assessment by multimodal fluorescence molecular imaging. Arterioscler Thromb Vasc Biol. 2012;32(11):2616-24.

14. Veilleux I, Spencer JA, Biss DP, et al. In Vivo Cell Tracking With Video Rate Multimodality Laser Scanning Microscopy. Selected Topics in Quantum Electronics, IEEE Journal of. 2008;14(1):10-8.

15. Schneider DJ, Sobel BE. A novel role for tissue-type plasminogen activator: prevention of thromboembolic occlusion. Circulation. 2008;118(14):1408-9.

16. McCarthy IR, Sazonova IY, Erdem SS, Hara T, Thompson BD, Patel P, et al. Multifunctional nanoagent for thrombus-targeted fibrinolytic therapy. Nanomedicine (Lond). 2012;7(7):1017-28.
17. Meyer BJ, Badimon JJ, Mailhac A, Fernandez-Ortiz A, Chesebro JH, Fuster V, et al. Inhibition of growth of thrombus on fresh mural thrombus. Targeting optimal therapy. Circulation. 1994;90(5):2432-8.

18. Pislaru SV, Pislaru $\mathrm{C}, \mathrm{Zhu} \mathrm{X}$, Arnout $\mathrm{J}$, Stassen $\mathrm{T}$, Vanhove $\mathrm{P}$, et al Comparison of a synthetic antithrombin III-binding pentasaccharide and standard heparin as an adjunct to coronary thrombolysis. Thromb Haemost. 1998;79(6):1130-5.

19. Ploen R, Sun L, Zhou W, Heitmeier S, Zorn M, Jenetzky E, et al. Rivaroxaban does not increase hemorrhage after thrombolysis in experimental ischemic stroke. J Cereb Blood Flow Metab. 2014;34(3):495-501.

20. Sun L, Zhou W, Ploen R, Heiland S, Zorn M, Veltkamp R. Rapid reversal of anticoagulation prevents excessive secondary hemorrhage after thrombolysis in a thromboembolic model in rats. Stroke. 2011;42(12):3524-9.

21. Zhu Y, Carmeliet P, Fay WP. Plasminogen activator inhibitor-1 is a major determinant of arterial thrombolysis resistance. Circulation. 1999;99(23):3050-5.

22. Yakovlev S, Makogonenko E, Kurochkina N, Nieuwenhuizen W, Ingham K, Medved L. Conversion of fibrinogen to fibrin: mechanism of exposure of tPAand plasminogen-binding sites. Biochemistry. 2000;39(51):15730-41.

23. Lord ST. Molecular Mechanisms Affecting Fibrin Structure and Stability. Arterioscler Thromb Vasc Biol. 2011;31(3):494-9.

24. Wohner N, Sotonyi P, Machovich R, Szabo L, Tenekedjiev K, Silva MM, et al. Lytic resistance of fibrin containing red blood cells. Arterioscler Thromb Vasc Biol. 2011;31(10):2306-13.

25. Jones G, Hunter F, Hancock HA, Kapoor A, Stone MJ, Wood BJ, et al. In vitro investigations into enhancement of tPA bioavailability in whole blood clots using pulsed-high intensity focused ultrasound exposures. IEEE Transactions on Biomedical Engineering.2010;57(1):33-6.

26. Marcos-Contreras OA, Ganguly K, Yamamoto A, Shlansky-Goldberg R, Cines DB, Muzykantov VR, et al. Clot penetration and retention by plasminogen activators promote fibrinolysis. Biochemical Pharmacology. 2013;85(2):216-22.

27. Henke PK, Pearce CG, Moaveni DM, Moore AJ, Lynch EM, Longo C, et al. Targeted deletion of CCR2 impairs deep vein thombosis resolution in a mouse model. J Immunol. 2006;177(5):3388-97.

28. Andia ME, Saha P, Jenkins J, Modarai B, Wiethoff AJ, Phinikaridou A, et al. Fibrin-targeted magnetic resonance imaging allows in vivo quantification of thrombus fibrin content and identifies thrombi amenable for thrombolysis. Arterioscler Thromb Vasc Biol. 2014;34(6):1193-8.

29. Vymazal J, Spuentrup E, Cardenas-Molina G, Wiethoff AJ, Hartmann MG, Caravan P, et al. Thrombus imaging with fibrin-specific gadolinium-based MR contrast agent EP-2104R: results of a phase II clinical study of feasibility. Invest Radiol. 2009;44(11):697-704.

30. Mackman N. New insights into the mechanisms of venous thrombosis. J Clin Invest. 2012;122(7):2331-6.

31. Jaffer FA, Calfon MA, Rosenthal A, Mallas G, Razansky RN, Mauskapf A, et al. Two-dimensional intravascular near-infrared fluorescence molecular imaging of inflammation in atherosclerosis and stent-induced vascular injury. J Am Coll Cardiol. 2011;57(25):2516-26.

32. Yoo H, Kim JW, Shishkov M, Namati E, Morse T, Shubochkin R, et al. Intra-arterial catheter for simultaneous microstructural and molecular imaging in vivo. Nat Med. 2011;17(12):1680-4.

33. Butte AN, Houng AK, Jang IK, Reed GL. Alpha 2-antiplasmin causes thrombi to resist fibrinolysis induced by tissue plasminogen activator in experimental pulmonary embolism. Circulation. 1997;95(7):1886-91. 